\title{
MARCH EGG NUMBER IN INBRED AND FIRST CROSS BROWN LEGHORNS
}

\author{
J. S. S. BLYTH \\ Poultry Research Centre, Edinburgh
}

IN the course of an earlier study (Blyth, 1952) the frequency distribution of pullets, on the basis of March egg numbers, was found to be negatively skewed, the curve approaching normality in shape for birds laying more than 18 eggs but tapering out in a long thin tail of less productive individuals. It was concluded that this resulted from the presence of two component types of variation--a basic maximum rate of laying (closely associated with egg weight), and irregularities superimposed upon it by gaps of varying length in the sequence of laying. There was some indication that the incidence of this pausing differed characteristically in the three inbred lines compared, and that it had increased between the two periods studied (1935-39 and 1945-49). The conjecture was also made there that pauses may depend on factors divorced from the direct process of egg formation. Lack of constitutional vigour might bring about such effects for, though difficult to pin down to particular causes, it must have its origin in shortcomings of development and function. Greenwood and Blyth (1948) have noted a tendency for different organ weaknesses to segregate in the isolate groups of the same Brown Leghorn flock; they inferred that the lowered mortality they encountered in first crosses might have resulted from the introduction of complementary desirable genes from the two parental lines, covering up each other's deficiencies.

While it cannot be assumed offhand that gaps in March production bear any relation to the well recognised interruptions of moult, broodiness and winter pause, it seems not unlikely that their incidence should reflect the birds' ability to withstand the more random environmental stresses that fall upon their ovarian activity throughout the year. Further examination of the March data available has therefore been made to learn how the variability in a successful interline cross compares with that in its parental types; whether, in fact, improvement in egg production comes about through a rise in rate, or reduction in irregularities in this rhythm, or both. Direct genetic effects on egg numbers could be expected to result in the $F_{1}$ mean being intermediate, or lying near that of one or other of the ancestral types, but it is unlikely that they would give rise to a decrease in pausing below that of the superior parental line; this would be a probable consequence however if degenerative deficiencies were influencing production as suggested above. 
The cross under discussion was made a number of times in different years; the annual progeny groups are small and their arrangement is unsuited to rigorous statistical comparisons; the mean value for each has therefore been presented separately to display the level of consistency among them. Of the two parental lines, one is a general purpose stock, (B), selected for a number of economic traits, while in the other, intensity (I), the emphasis has been solely on intensity of production. March records for both are described in the original publication. Information about the numbers of birds in the subsequent comparisons, and the years in which they were hatched, are given in table I; in designating the reciprocal matings the initial

TABLE I

Number of puliets per group

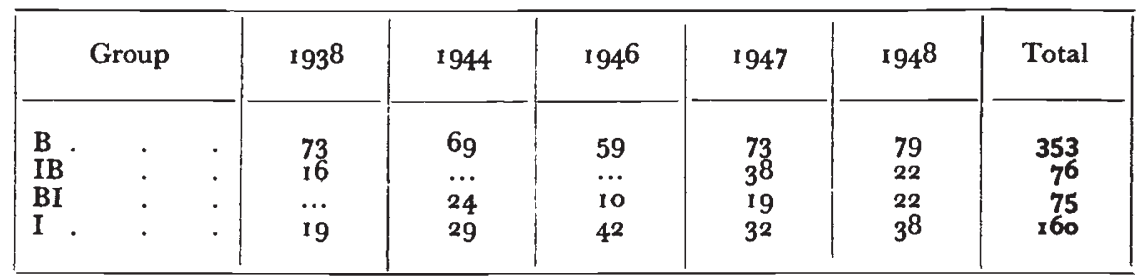

letter of the dam's line is given first. All the annual samples of the crosses were from single pen matings except the small 1946 one which derived from two cocks; the average yearly number of cocks used in the lines was four for B and two for I. A more general comparison of traits in these crosses and the participant lines has been made by Greenwood and Blyth (1951).

Rate.-The method of exposing differences in rate used before was to compare groups after eliminating birds falling below certain levels of production (18, 22, or 24 eggs). Such a measure is obviously only approximate but the reliability of possible alternatives is equally unknown so it is the first procedure used again here, with 22 eggs as the lower limit. In table 2 the mean egg number for this class is contrasted with that for all pullets in the samples of reciprocal first crosses and the representatives of their parental lines hatched in the same years. The general means for the complete populations indicate superiority of the reciprocals over both parent types, and only in one of the separate samples does this not hold true : there, in 1938 , the cross had a B sire (IB) and falls in an intermediate position. Among the curtailed classes (table $2 \mathrm{~B}$ ) the higher position of the crosses is no longer maintained: they still exceed $B$ though the range of variation is now smaller, but are indistinguishable from $I$ in performance.

The difference in the levels of the corresponding means in the two tables is not necessarily wholly accounted for by the inclusion in the first of birds with production gaps : pausing individuals may 
also be intermingled with non-pausing higher producers. It was the supposition that this occurred that led to placing the arbitrary line of division so high. Such an arrangement of course has the concomitant disadvantage that it may be eliminating too large a proportion of pullets which are laying at a low basic rate, and so unbalancing some of the frequency distributions. It is quite conceivable, for instance, that inherent rates yielding less than 22 eggs in the month occur : a bird laying every other day could only produce I 5 or 16 eggs ; on the other hand birds with high potential rates could miss several consecutive days without falling below the limit

TABLE 2

Mean number of eggs in March

\begin{tabular}{|c|c|c|c|c|c|c|c|}
\hline & Group & r 938 & r944 & 1946 & I 947 & 1948 & Total \\
\hline \multicolumn{8}{|c|}{ 2a. All Birds } \\
\hline $\begin{array}{l}\text { B } \\
\text { IB } \\
\text { BI } \\
\text { I }\end{array}$ & $:$ & $\begin{array}{c}21 \cdot 0 \\
23 \cdot 2 \\
\ldots \\
24 \cdot 0\end{array}$ & $\begin{array}{c}22 \cdot I \\
\ldots \\
24 \cdot 7 \\
24 \cdot 1\end{array}$ & $\begin{array}{c}15.6 \\
\ldots \\
21 \cdot 2 \\
19.6\end{array}$ & $\begin{array}{l}23 \cdot 1 \\
24 \cdot 4 \\
24 \cdot 3 \\
23 \cdot 3\end{array}$ & $\begin{array}{l}19 \cdot 3 \\
25 \cdot 1 \\
22 \cdot 5 \\
22 \cdot 0\end{array}$ & $\begin{array}{l}20 \cdot 35 \\
24 \cdot 33^{*} \\
23 \cdot 47^{*} \\
22 \cdot 27^{*}\end{array}$ \\
\hline \multicolumn{8}{|c|}{ 2b. Birds Laying 22 or more } \\
\hline $\begin{array}{l}\text { B } \\
\text { IB } \\
\text { BI } \\
\text { I }\end{array}$ & i. & $\begin{array}{c}23 \cdot 2 \\
23 \cdot 4 \\
\ldots \\
25 \cdot 2\end{array}$ & $\begin{array}{c}23 \cdot 7 \\
\ldots . \\
24.9 \\
25.0\end{array}$ & $\begin{array}{c}22 \cdot 9 \\
\ldots \\
25 \cdot 3 \\
24 \cdot 5\end{array}$ & $\begin{array}{l}24 \cdot 5 \\
25 \cdot 1 \\
25 \cdot 4 \\
25 \cdot 1\end{array}$ & $\begin{array}{l}23 \cdot 6 \\
25.4 \\
23 \cdot 7 \\
24.9\end{array}$ & $\begin{array}{l}23 \cdot 80 \\
24^{\cdot} 82^{*} \\
24^{\prime} 7^{*} \\
24^{*} 94^{*}\end{array}$ \\
\hline
\end{tabular}

* $t$ test for correspondence with B mean has a probability $<0 \cdot 001$.

adopted. In the first case there would be an overestimate of the mean in groups which contained many individuals with a genetically low rate of laying, while the second contingency could act in the opposite direction. The only test of this which it seemed worthwhile undertaking on the present restricted data was that made by eliminating all birds with gaps greater than a single day. This classification may be affected by the distribution of unrecorded (floor) eggs but these did not exceed 2 per cent. of the total, were usually below I per cent., and did not appear to be abnormally distributed among the samples.

When the means were recalculated in this way they differed little from those for the 22-up samples except in B groups where the difference was always negative, and resulted in a decrease of about half an egg in its general mean to 23.14 eggs; the comparable figures for the others were, IB, $24 \cdot 98$; BI, $24 \cdot 75$; and I, $24 \cdot 84$ eggs, and their individual year samples all differed significantly from corresponding B means except I I 947 and BI 1948. This suggests that while the first method of determining basic rate was satisfactory for 
the last three groups, it eliminated more B birds which laid at a naturally low rate, and so overestimated the mean there.

The proportion of pullets in a sample which lay less than 22 eggs (table 3) also demonstrates that B differs from the others but again does not distinguish between variations due to rate and those in the

TABLE 3

Percentage laying less than 22 eggs

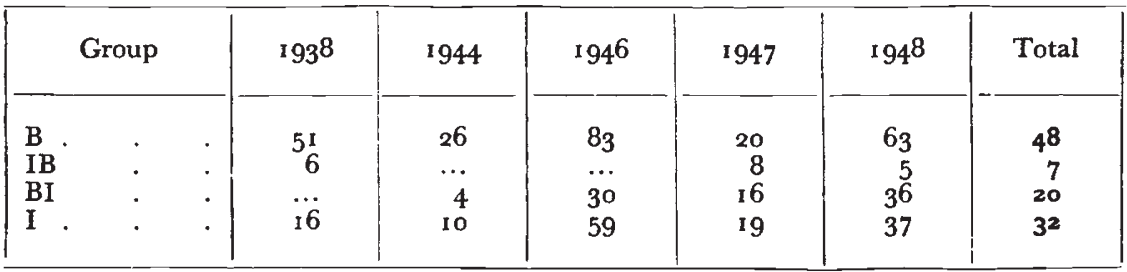

incidence of deviations from it; half the $B$ birds fall below the arbitrary division line ; only about a third of the I group do so, and still less of the crosses. As there are only two direct comparisons available between the reciprocal samples (1947 and 1948) the tendency for IB to rank slightly better than BI on mean performance, which manifests itself in this and the following table, will merely be noted here without comment.

Pausing.-Non-genetic fluctuations in the basic maximum rate may occur both seasonally and in adverse environments such as a late snowstorm encountered by 1946 hatched pullets in the following

TABLE $4 \mathrm{~A}$

Percentage with gaps over I day

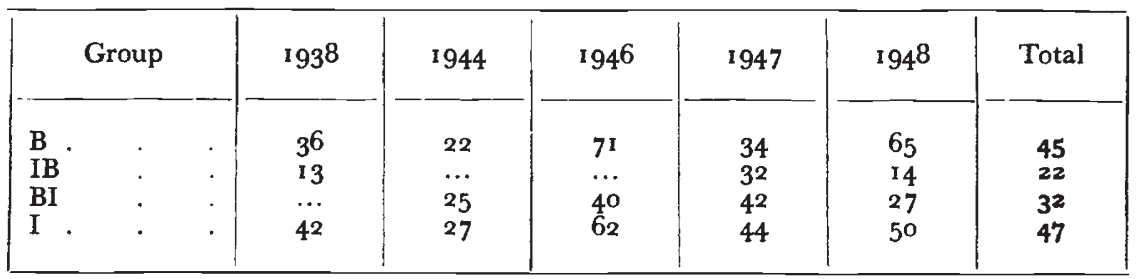

March. These as well as unrecorded eggs might upset calculations of the number of missed potential laying days. The proportions of pausing birds therefore have been set out for two levels : those with maximum gaps of over one day, and over four days, irrespective of the number of eggs they lay. In table $4 \mathrm{~A}$ the first of these classifications, while showing an overall smaller proportion of crosses with gaps exceeding a single day, does not differentiate very well between them and the inbreds within particular years. In table $4 \mathrm{~B}$, however, the absence of longer pauses in the former is more striking; in actual numbers, only one IB and three BI birds stopped for more than 
four days while $29 \mathrm{I}$ and $66 \mathrm{~B}$ did so. Comparison of the two halves of the table gives the impression that there may be no real difference between the crosses and the lines in regard to smaller interruptions and that it is mainly the incidence of larger ones that is affecting the annual production average.

TABLE 4 B

Perrentage with gaps over 4 days

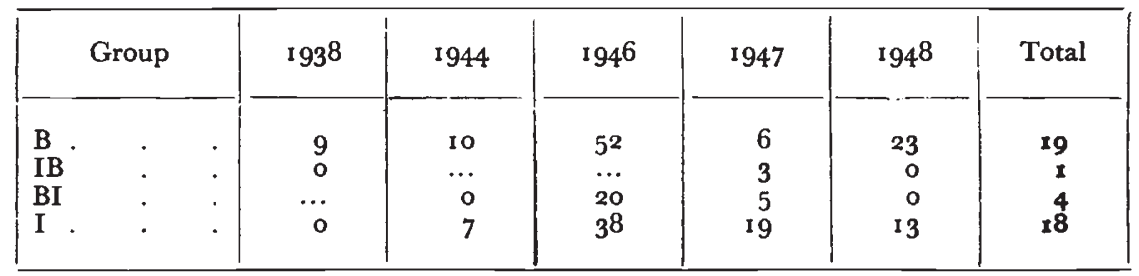

A new point emerges here about the two inbred lines; though relatively fewer I than $\mathrm{B}$ pullets were found to fall below the 22 egg limit (table 3), the percentage of long and short pauses is roughly the same in both. This casts doubt on the inference drawn earlier that there may be characteristic differences in the incidence of pausing between lines; it can now be seen that it may have arisen because relatively more non-pausing birds were being included in the under 22-egg class for one line while some were pausing in another without falling below the 22-egg limit. As the same source of confusion may be operating in the interperiod difference which was reported for I 935-39 and I 945-49 (Blyth, I952), a re-check of all March data for the two lines has been made. There were I5 B and II I annual samples considered of sufficient size for examination (I9 pullets and over). When the birds with greater than single day gaps were eliminated the difference between the estimate for mean rate of production from this and from the 22-up classification was 0.62 eggs with a standard error of 0.10 for $B$, and $-0.06 \pm 0.10$ for $\mathrm{I}$ in favour of the latter technique of measurement ; this corresponds well with the observations made on the restricted data and confirms the view that rate in the $\mathrm{B}$ line had been consistently overestimated.

The situation with regard to changes in the proportion of gaps between the two half decades is demonstrated in table 5. For both lines there is a significant increase in the number of birds with gaps over 4 days between pre- and post-war periods $\left(\mathrm{Chi}^{2}=9, d f \mathrm{r}\right.$, in each case); when the worst year (1946) is excluded the difference is no longer statistically significant but it is still in the same direction. Two to four day gaps in the B line also behave in the same way, but in $\mathrm{I}$ it is in the earlier period that small irregularities are more frequent, and so much so that when the two classifications are added together the two periods are practically alike in their proportions of pausing birds. This discrepancy between the two lines however may be more apparent than real: if there is a tendency towards 
greater divergence from the potential maximum rate in more recent times this could occur in high producers as the absence of single interclutch eggs, and would go undetected into the estimate of rate, but in pullets in which the rate of laying was not far from the 50 per cent. level it is manifestly impossible for interruptions to happen without bringing them into the categories classifying pausing birds. Thus a slight shift towards more irregularities in I might not be evident in birds functioning close to their maximum because their high basic rate would hide this weakening of their ability ; it would be mainly the proportion already showing gaps which could measurably express this deterioration. In the $\mathrm{B}$ line with its lower average rate, more birds would be near the critical level where any downward trend would be recognisable with the criteria used. On this assumption,

TABLE 5

Percentage of maximum gaps

\begin{tabular}{|l|c|c|c|c|c|c|}
\hline & \multicolumn{3}{|c|}{ B } & \multicolumn{3}{|c|}{ I } \\
\cline { 2 - 7 } & $\begin{array}{c}\text { No. of } \\
\text { birds }\end{array}$ & $\begin{array}{c}2-4 \\
\text { days }\end{array}$ & $\begin{array}{c}\text { Over } \\
\text { 4 days }\end{array}$ & $\begin{array}{c}\text { No. of } \\
\text { birds }\end{array}$ & $\begin{array}{c}2-4 \\
\text { days }\end{array}$ & $\begin{array}{c}\text { Over } \\
\text { 4 days }\end{array}$ \\
\hline & 305 & 24 & 11 & 143 & 34 & 7 \\
\hline $1935-40^{*}$ & 39 & 28 & 20 & 185 & 25 & 19 \\
$1945-49$. & 397 & 28 & 143 & 26 & 13 \\
\hline do. excl. 1946 & 338 & 30 & 14 & 143 & \\
\hline
\end{tabular}

* 1937 data excluded because there were too few I birds.

among pullets described in table 5, B birds with gaps of four days have increased in number in the more recent period at the expense of those showing two to four day gaps, while their ranks in turn have been more than replenished by a decrease in the number of birds with no classifiable interruptions. In $I$, the latter transfer is not occurring to a detectable extent.

\section{DISCUSSION}

The advantages of separating basic rate of laying from pauses in it for the interpretation of variations in production are again evident here. That heterotic effects in the first crosses studied were restricted to reduction of pausing, and were not detectable in basic rate, is a point of considerable interest which merits further investigation. In particular, the question arises whether it is a phenomenon of general validity for the trait, and occurs in breed crosses which are superior to their parental types, or if it is confined to the field of interline matings where crossing overcomes inbreeding decay.

The earlier idea (Blyth, 1952) that there were differences in the incidence of pausing birds in the inbred lines is not substantiated by 
the present analysis; it now appears to have arisen because the original inference was drawn from data in which the criteria used did not sufficiently differentiate between pullets with a low basic rhythm and those with actual interruptions in production. The weight of evidence however is still in favour of the view that there has been a slight increase in the proportion of pausing birds between the two five-year periods, 1935-39 and 1945-49, accompanying the increase in basic rate found there. This, and the greater ability of the crosses to resist interruptions in laying while not exceeding the better parental line in potential rate, point to the connection between these two components of production being of an indirect nature.

The corollary of such an assumption is that the major, or majority of, deficiencies must be complementary in the two lines, so they provide no evident reason for equality in the degree of pausing. It may be that the effective basis of degeneration takes the form of a multiplicity of small, often non-specific, genetic changes, which while increasing genetic variability, yield a greater uniformity of phenotypic expression in the lines. This would be in accord with the lack of exhaustion of genetic variation found by Lerner and Dempster (I95I) in highly selected stocks and discussed by them in relation to genetic homeostasis.

The distinction between fluctuations in basic maximum rate and digressions from it may be purely arbitrary in practice but the facts lead to the conclusion that they have separate fundamental sources. Apart from any importance that this would have in the formulation of selection indexes for the more rapid improvement of flocks $(v$. Lerner, I950), it might come to be of considerable technical value. In particular it gives hope of some relief from the labour of trapnesting, for although accurate recording must remain a prerequisite of estimations of basic rate, it is only necessary for a restricted period.

As to pausing, the present study points to the genetic unimportance of the smaller interruptions in laying so that, for comparisons on this basis, less detailed information may prove just as satisfactory as fulltime trapping. Reports on the relative reliability of a number of techniques of intermittent and part-time recording are available (Dudley, I93 I ; Norskog, I $94^{8}$; Read, I949) but concrete suggestions as to which would be best adapted to gaining selective control over this aspect of production must await further investigation. Winter pause, moult, and in some breeds, broodiness, may continue to require separate attention, but differences between birds in respect of the " non-specific" pausing encountered here may be identifiable at certain seasons without recourse to observation of the entire annual cycle. Again, if pausing, as distinct from fluctuations in rate, involves a definite cessation and re-initiation of the ovulatory rhythm, it could probably be identified sufficiently accurately by routine observation of comb condition in small flocks where birds are marked with large numbered visible wing tabs; in larger commercial projects however 
this would probably offer more technical difficulties than procedures like 5-day-week trapnesting or whatever other objective measurement was found to fulfil requirements.

Finally, because of the loss of information which must result from confusing the effects of their variations with those of basic rate when pullet production is evaluated from a single figure, the possibility of measuring pausing of all types from annual records by correcting the latter for rate is also one that might repay serious consideration.

\section{SUMMARY}

An analysis of the March production in a small number of replications of an interline cross indicates that its superiority over the parental types rest on two kinds of variations in egg numbers, $(a)$ a basic maximum rate or rhythm, and $(b)$ gaps constituting divergencies from it. The basic rate manifested equalled, but did not exceed, that of the higher producing line. The proportion of cross birds pausing for more than four days is very small and provides a source of distinction from both inbreds.

A previous deduction that there were differences in the incidence of pausing between inbred lines now appears to have originated in an artifact arising from the criterion of classification used, but reexamination of the data still suggests that the proportion of pausing birds in them has increased in recent years.

It is concluded that the two types of variation distinguished have separate and independent origins. The possible implications of this in regard to practical methods of recording egg production for selection purposes are discussed.

\section{REFERENCES}

BLYTH, J. S. S. 1952. The correlation between egg number and egg weight in the fowl ; an investigation of its inconstancy. Poult. Sci., 3I, 254-268.

DUDLEY, F. J. 1931. Short period trapnesting as a means of estimating annual egg production and average annual weight. Harper Adams Util. Poult. F., I6, 557-562.

GREENWOOD, A. W., AND BLYTH, J. s. s. 1948. The problem of pullet mortality. Official Report 8th World's Poult. Congrcss, Copenhagen, pp. 687-69i.

GREENWOOD, A. W., AND BLYTH, J. S. S. 1951. A repeated cross between inbred lines. F. Agric. Sci., 4I, 367-37o.

Lerner, I. M. 1950. Population Genetics and Animal Improvement. Cambridge Univ. Press. $x v i i i+342$ pp.

LERNER, I. M., AND DEMPSTER, E. K. 1951. Attenuation of genetic progress under continued selection in poultry. Heredity, 5, 75-94.

Norskor, A. W. 1948. Periodical trapnesting and family selection for egg production. Poult. Sci., 27, 71 3-718.

READ, D. R. 1949. A study of the accuracy of simple sampling methods for the estimation of egg production and mean egg weight. F. Agric. Sci., 39, 259-264. 